\title{
NATURAL PRECONDITIONING AND ITERATIVE METHODS FOR SADDLE POINT SYSTEMS
}

\author{
JENNIFER PESTANA AND ANDREW J. WATHEN
}

\begin{abstract}
The solution of quadratic or locally quadratic extremum problems subject to linear(ized) constraints gives rise to linear systems in saddle point form. This is true whether in the continuous or discrete setting, so saddle point systems arising from the discretization of partial differential equation problems, such as those describing electromagnetic problems or incompressible flow, lead to equations with this structure as do, for example, interior point methods and the sequential quadratic programming approach to nonlinear optimization.

This survey concerns iterative solution methods for these problems and in particular shows how the problem formulation leads to natural preconditioners which guarantee a fast rate of convergence of the relevant iterative methods. These preconditioners are related to the original extremum problem and their effectiveness - in terms of rapidity of convergence - is established here via a proof of general bounds on the eigenvalues of the preconditioned saddle point matrix on which iteration convergence depends.
\end{abstract}

Key words. inf-sup constant, iterative solvers, preconditioning, saddle point problems

1. Introduction. When a quadratic functional is minimized subject to linear(ized) constraints, Lagrange multipliers and stationarity conditions lead to saddle point systems. When constraints are not exactly enforced or when a penalty, regularization or stabilization term is included, we obtain a generalized saddle point problem. Both standard and generalized saddle point problems are ubiquitous in scientific computing, with important applications including electromagnetics, incompressible fluid dynamics, structural mechanics, constrained and weighted least squares, constrained optimization, economics, interpolation of scattered data, model order reduction and optimal control [7, Section 2]. Thus, the numerical solution of standard and generalized saddle point problems, which we call saddle point problems for brevity, are of significant interest.

Certain saddle point problems are discrete in nature and lead directly to a linear system. In other applications, the original problem is continuous and must be discretized if it is to be solved numerically. In either case to obtain the solution to the saddle point problem (or an approximation to it in the case of discretization) we must solve the linear system

$$
\underbrace{\left[\begin{array}{cc}
A & B^{T} \\
B & -C
\end{array}\right]}_{\mathcal{A}}\left[\begin{array}{l}
u \\
p
\end{array}\right]=\left[\begin{array}{l}
f \\
g
\end{array}\right],
$$

where $A \in \mathbb{R}^{n \times n}$ is symmetric and is positive definite on the nullspace of $B, B \in$ $\mathbb{R}^{m \times n}, m \leq n$ and $C \in \mathbb{R}^{m \times m}$ is symmetric positive semidefinite. Note that $C$ is the zero matrix in a standard saddle point system. The vector $u$ is associated with the primal variables while $p$ may be viewed as the Lagrange multipliers. In optimization, the system (1.1) is related to first-order stationarity conditions of the equality constrained quadratic program

$$
u=\arg \min _{v} \frac{1}{2} v^{T} A v-f^{T} v \text { subject to } B v=g .
$$

To highlight how frequently saddle point systems appear, and their connection to constrained minimization, let us discuss three simple applications in addition to 
the quadratic program (1.2) above. The first is the solution of Stokes equations in incompressible fluid dynamics, the second is related to interpolation on the sphere by a hybrid method, while the third describes the flow of current in a closed electric circuit.

In their simplest form, the equations governing the velocity $u \in \mathbb{R}^{d}, d=2,3$, and pressure $p \in \mathbb{R}$ of a Stokes fluid in a bounded connected domain $\Omega$ with piecewise smooth boundary $\partial \Omega=\partial \Omega_{D} \cup \partial \Omega_{N}$ are [27, Chapter 5], [25, Chapter 3]:

$$
-\nabla^{2} u+\nabla p=0, \quad \nabla \cdot u=0 \text { in } \Omega, \quad u=0 \text { on } \partial \Omega_{D}, \quad \frac{\partial u}{\partial n}-n p=s \text { on } \partial \Omega_{N},
$$

where $n$ is the outward-pointing unit normal vector to the boundary and $\partial u / \partial n$ is the directional derivative of the velocity in the unit normal direction. The Stokes equations are a continuous saddle point problem in which the velocity $u$ is the primal variable and the pressure $p$ is the Lagrange multiplier. Associated with this system we should expect to find a variational problem and indeed, the Stokes equations minimize the viscous energy subject to the incompressibility constraint and boundary conditions.

Saddle point systems also arise when finding interpolants on a sphere $\mathbb{S}^{d} \subset \mathbb{R}^{d+1}$ by hybrid methods that combine local radial basis functions with global spherical polynomials $[63,70]$. To ensure that the interpolant is unique, the radial basis function component is constrained to be orthogonal to the spherical polynomial approximation space with respect to the inner product associated with the radial basis functions (the native space inner product). Thus, the associated extremum problem is to minimize the interpolation error subject to this orthogonality constraint. To compute the interpolant we solve a saddle point system for the coefficients of the radial basis function interpolant (the primal variables) and the spherical harmonic interpolant (the Lagrange multipliers).

Computing the flow of current in a closed electric circuit using Ohm's Law and Kirchoff's current and voltage laws leads to an inherently discrete saddle point system for the potentials $v$ (the primal variables) and currents $i$ (the Lagrange multipliers) [54], [65, Section 2.3], [66]. The related variational problem is the minimization of potential energy subject to conservation of charge.

These applications raise two important points. The first is that many physical systems lead to saddle point systems, since we are frequently interested in minimizing some energy functional subject to the conservation of a physical quantity. The second is that the Lagrange multipliers are often important, either for checking optimality or because they have physical relevance. Indeed, in the hybrid approximation application the interpolant cannot be recovered without the Lagrange multipliers. An advantage of the saddle point system is that the primal variables and Lagrange multipliers are simultaneously computed.

When (1.1) is large and sparse, iterative methods such as Krylov subspace methods are particularly attractive. However, the rate of convergence of these methods depends on the spectrum of the saddle point matrix - since it is real symmetric - and may be very slow. One issue is the indefiniteness of saddle point systems, which can delay the speed of convergence of Krylov methods. Additionally, ab initio discrete problems may have poor spectra while problems that involve discretization usually have eigenvalues that vary with the discrete approximation.

These, and other, causes of slow convergence rates are typically remedied by preconditioning. We focus here on block diagonal preconditioners but note that many 
preconditioners for saddle point matrices have been proposed, such as block triangular [12, 40, 42, 49], constraint [22, 23, 41, 46], augmented Lagrangian [9, 34] and splitting-based $[6,62]$ preconditioners. For more details we refer to Benzi, Golub and Liesen [7].

Many issues must be addressed when solving saddle point problems, including whether the problem has a unique solution, how to discretize a continuous saddle point problem, how to choose an appropriate preconditioner and how to predict the rate of convergence that can be expected from the preconditioned iterative method. It is somewhat surprising that for certain block diagonal preconditioners the answers to all of these questions are linked. Another issue that arises in optimization (for example in SQP solvers) is that the solution to the saddle point system (1.1) should also give sufficient descent of a merit function. Descent directions are beyond the scope of this survey but we refer the interested reader to the discussion of Byrd, Curtis and Nocedal [17].

It is the aim of this survey to give an overview of the solution process for all saddle point problems, regardless of the particular application. We give conditions under which the original saddle point formulation is solvable and, if discretization is required, state an additional condition for the resulting finite dimensional problem. These conditions lead to "natural" block diagonal preconditioners and allow bounds on the eigenvalues of the preconditioned matrix to be determined that can be used to estimate the speed of convergence of iterative methods applied to the resulting preconditioned system. Although the focus here is on iterative methods, these eigenvalue bounds are also important when the saddle point system (1.1) is solved by direct methods since for the (typically unpreconditioned) problem, the condition number can affect the accuracy of the computed solution.

A wealth of literature on solving saddle point systems exists, much of it related to particular applications. Perhaps the most comprehensive work is the survey by Benzi, Golub and Liesen [7], which considers conditions under which the linear system (1.1) is solvable and block diagonal preconditioners but which does not discuss in great detail the underlying (possibly infinite dimensional) saddle point problem. The conditions for a unique solution (often called second-order sufficient conditions) are often discussed and can be found in, for example, the monograph by Nocedal and Wright [50] or, in the substantial area of PDEs, in Babuška [4] and Brezzi [14].

The "natural" or norm-based block diagonal preconditioners we describe have been studied, largely in the context of PDEs, by Elman, Silvester and Wathen [27, Chapter 6] and [25, Chapter 4], Hiptmair [38], Loghin and Wathen [45], Mardal and Winther [47], Vassilevski [68] and Zulehner [79]. Related preconditioners that are based only on a study of the eigenvalues of the preconditioned matrix have also been proposed [40, 43, 49]. Norm-based preconditioning also arises in particular applications in PDEs, such as in groundwater flow [12, 13, 21, 33, 55, 69], Stokes flow $[18,20,76,59,74]$, elasticity $[2,16,32,42,60]$, magnetostatics $[52,53]$ and in the hybrid interpolation scheme on the sphere [44]. We note that Arioli and Loghin [1] also use norm equivalence to investigate appropriate stopping criteria for iterative methods applied to mixed finite element problems.

Eigenvalue bounds for block diagonal preconditioned saddle point matrices are also prevalent in the literature. When the finite dimensional saddle point matrix is a standard saddle point problem the bounds of Rusten and Winther [55] can be used. For generalized saddle point matrices, Elman, Silvester and Wathen [27, 25], Axelsson and Neytcheva [3] and Gould and Simoncini [36] have bounds that may be applied. 
The rest of this survey aims to unify this saddle point theory in an applicationindependent manner. For simplicity of exposition most of our results are given for saddle point problems in which $A$ is positive definite and we defer the discussion of saddle point systems in which $A$ is positive definite on the nullspace of $B$ to the end of the manuscript. In Section 2 we briefly describe saddle point problems for which $A$ is positive definite and give conditions under which a unique solution exists. We discuss the discretization of continuous problems and the extra conditions that must be imposed on the discrete problem to guarantee a unique solution. In Section 3 we describe the corresponding linear system that must be solved and show that the conditions for a unique solution have linear algebra analogues that are useful when considering natural preconditioners and convergence bounds. Section 4 discusses the Krylov subspace methods that may be applied to these saddle point problems and describes natural block diagonal preconditioners, while bounds on the speed of convergence of the Krylov method MINRES [51] applied to the block diagonal preconditioned Krylov method are given in Section 5. We apply the results of previous sections to the Stokes, hybrid interpolation and circuit problems in Section 6 and in Section 7 we discuss extensions of the results in previous sections to problems with matrices $A$ that are semidefinite, but positive definite on the nullspace of $B$. Our conclusions are given in Section 8.

Throughout, we let $x^{T}$ denote the transpose of the vector $x$ and similarly for matrices. We use the Löwner ordering for symmetric matrices so that matrices $M_{1}, M_{2} \in \mathbb{R}^{p \times p}$ satisfy $M_{1} \succ M_{2}\left(M_{1} \succeq M_{2}\right)$ if and only if $M_{1}-M_{2}$ is symmetric positive definite (semidefinite).

2. Saddle point formulations. In this section we show how the saddle point formulation with $A$ positive definite is obtained from a variational form with constraints, discuss generalized saddle point problems, and give conditions under which a unique solution to the standard and generalized saddle point problems exist.

2.1. Saddle point systems. Here we introduce a general framework for saddle point problems. Since some applications, such as those involving partial differential equations, result in infinite dimensional problems, we utilize bilinear forms in Hilbert spaces. However, we stress that the framework is equally applicable to problems that are ab initio discrete.

Consider two real Hilbert spaces $\mathcal{X}$ and $\mathcal{M}$ with dual spaces $\mathcal{X}^{\prime}$ and $\mathcal{M}^{\prime}$ and let $a: \mathcal{X} \times \mathcal{X} \rightarrow \mathbb{R}, b: \mathcal{X} \times \mathcal{M} \rightarrow \mathbb{R}$ be bounded bilinear forms with

$$
\begin{gathered}
|a(v, w)| \leq \Gamma_{a}\|v\|_{\mathcal{X}}\|w\|_{\mathcal{X}} \text { for all } v, w \in \mathcal{X}, \\
|b(v, q)| \leq \Gamma_{b}\|v\|_{\mathcal{X}}\|q\|_{\mathcal{M}} \text { for all } v \in \mathcal{X}, q \in \mathcal{M} .
\end{gathered}
$$

Let us additionally assume that $a$ is symmetric and coercive on $\mathcal{X}$ (X्X-elliptic) so that $a(v, w)=a(w, v)$ for all $v, w \in \mathcal{X}$ and there exists some positive constant $\alpha$ for which $a(v, v) \geq \alpha\|v\|_{\mathcal{X}}^{2}$ for all $v \in \mathcal{X}$. The norms $\|\cdot\|_{\mathcal{X}}$ and $\|\cdot\|_{\mathcal{M}}$ are defined in the usual way in terms of the Hilbert space inner products $(\cdot, \cdot)_{\mathcal{X}}$ and $(\cdot, \cdot)_{\mathcal{M}}$. We stress that the Hilbert spaces may have finite or infinite dimension; the latter case is common in, for example, problems arising from partial differential equations while the former situation arises in, for example, optimization where $\mathcal{X}=\mathbb{R}^{n}$ and $\mathcal{M}=\mathbb{R}^{m}$ with $n \geq m$.

The variational problem we consider is to find

$$
u=\arg \min _{v \in \mathcal{X}} J(v)=\frac{1}{2} a(v, v)-f(v) \text { such that } b(v, q)=g(q) \text { for all } q \in \mathcal{M} \text {, }
$$


where $f \in \mathcal{X}^{\prime}$ and $g \in \mathcal{M}^{\prime}$ are bounded linear functionals. Note that for the quadratic program the constraints in (2.3), namely $q^{T}(B v-g)=0$ for all $q \in \mathbb{R}^{m}$, are equivalent to the constraints $B v=g$ in (1.2)

Rather than solving the variational problem (2.3) directly, we can formulate an equivalent saddle point system by first introducing the Lagrange function

$$
\mathcal{L}(v, q)=J(v)+b(v, q)-g(q), v \in \mathcal{X}, q \in \mathcal{M},
$$

which coincides with $J$ when the constraints are satisfied. The first-order stationarity conditions for the Lagrange function are satisfied if we find $(u, p) \in \mathcal{X} \times \mathcal{M}$ such that

$$
\begin{aligned}
a(u, v)+b(v, p) & =f(v) \text { for all } v \in \mathcal{X}, \\
b(u, q) & =g(q) \text { for all } q \in \mathcal{M} .
\end{aligned}
$$

The saddle point system (2.4) is so named because any solution $(u, p)$ also satisfies the saddle point property $\mathcal{L}(u, q) \leq \mathcal{L}(u, p) \leq \mathcal{L}(v, p)$ for all $(v, q) \in \mathcal{X} \times \mathcal{M}$ and, crucially, $u$ is a minimizer of (2.3). Note that for the quadratic program (1.2), the equations (2.4) become

$$
v^{T}\left(A u+B^{T} p-f\right)=0 \text { for all } v \in \mathbb{R}^{n}, \quad q^{T}(B u-g)=0 \text { for all } q \in \mathbb{R}^{m}
$$

and are equivalent to the conditions $A u+B^{T} p=f, B u=g$. This shows that (2.4) is a variational form of the standard first-order conditions, with $p$ the Lagrange multiplier.

An infinite-dimensional saddle point example is provided by the Stokes equations introduced in Section 1. Multiplying (1.3) by an appropriate test function and integrating by parts leads to the standard weak formulation: find $(u, p) \in H_{0}^{1} \times L_{2}(\Omega)$ such that

$$
\int_{\Omega} \nabla u: \nabla v-\int_{\Omega} p \nabla \cdot v=\int_{\partial \Omega_{N}} s \cdot v \text { for all } v \in H_{0}^{1}, \quad \int_{\Omega} q \nabla \cdot u=0 \text { for all } q \in \mathrm{L}_{2}(\Omega),
$$

where $H_{0}^{1}=\left\{v \in \mathcal{H}^{1}(\Omega)^{d}: u=0\right.$ on $\left.\partial \Omega_{D}\right\}, \mathcal{H}^{1}(\Omega)^{d}$ is the standard Sobolev space of vector-valued functions with each component having square integrable (weak) derivatives in $\Omega$, and $\nabla u: \nabla v$ represents a component-wise scalar product. For further details we refer the reader to Elman, Silvester and Wathen [27, Chapter 5] or [25, Chapter 3]. Thus, in this setting $\mathcal{X}=H_{0}^{1}, \mathcal{M}=L_{2}(\Omega)$,

$$
a(u, v)=\int_{\Omega} \nabla u: \nabla v
$$

is the viscous energy and

$$
b(u, q)=\int_{\Omega} q \nabla \cdot u .
$$

To this point, we have assumed that the constraint (2.3) is exactly satisfied. However, there may be reasons to relax this constraint or to apply a penalty term. Examples include nearly incompressible fluid flow [11, Chapter III, Sections 4 and 6], the regularization of an ill-posed problem [8] or certain interior point methods [77, 78]. In these cases we obtain the generalized saddle point system: find $(u, p) \in \mathcal{X} \times \mathcal{M}$ such that

$$
\begin{aligned}
& a(u, v)+b(v, p)=f(v) \text { for all } v \in \mathcal{X}, \\
& b(u, q)-c(p, q)=g(q) \text { for all } q \in \mathcal{M} .
\end{aligned}
$$


Here $c: \mathcal{M} \times \mathcal{M} \rightarrow \mathbb{R}$ is a third bilinear form that is assumed to be bounded, symmetric and nonnegative on $M$, so that

$$
|c(r, q)| \leq \Gamma_{c}\|r\|_{\mathcal{M}}\|q\|_{\mathcal{M}}, \text { for all } r, q \in \mathcal{M},
$$

$c(r, q)=c(q, r)$ and $c(q, q) \geq 0$ for all $r, q \in \mathcal{M}$. Associated with this generalized saddle point problem (2.6) is the functional

$$
\mathcal{L}_{c}(v, q)=\frac{1}{2} a(v, v)-f(u)+b(v, q)-\frac{1}{2} c(q, q)-g(q), v \in \mathcal{X}, q \in \mathcal{M},
$$

and, analogously to the standard problem, solutions $(u, p)$ of $(2.6)$ satisfy the saddle point property $\mathcal{L}_{c}(u, q) \leq \mathcal{L}_{c}(u, p) \leq \mathcal{L}_{c}(v, p)$ for all $(v, q) \in \mathcal{X} \times \mathcal{M}$

Clearly, (2.4) is obtained from (2.6) by setting $c \equiv 0$. Consequently, the conditions under which both problems are uniquely solvable can be described by a single result [14], [79, Theorem 2.6]:

TheOrEm 2.1. Let $\mathcal{X}$ and $\mathcal{M}$ be real Hilbert spaces with norms $\|\cdot\| \mathcal{X}$ and $\|\cdot\|_{\mathcal{M}}$ induced by inner products $(\cdot, \cdot)_{\mathcal{X}}$ and $(\cdot, \cdot)_{\mathcal{M}}$. Let $a: \mathcal{X} \times \mathcal{X} \rightarrow \mathbb{R}, b: \mathcal{X} \times \mathcal{M} \rightarrow \mathbb{R}$ and $c: \mathcal{M} \times \mathcal{M}: \rightarrow \mathbb{R}$ be bilinear forms that satisfy (2.1), (2.2) and (2.7), respectively, and let $a$ and $c$ be symmetric. Let $f: \mathcal{X} \rightarrow \mathbb{R}$ and $g: \mathcal{M} \rightarrow \mathbb{R}$ be bounded linear functionals on $\mathcal{X}$ and $\mathcal{M}$, respectively. Then if

$$
c(q, q) \geq 0 \text { for all } q \in \mathcal{M},
$$

and if there exists a constant $\alpha>0$ such that

$$
a(v, v) \geq \alpha\|v\|_{\mathcal{X}}^{2}, \quad \text { for all } v \in \mathcal{X},
$$

and if there exists a constant $\beta>0$ such that the inf-sup condition

$$
\sup _{v \in \mathcal{X} \backslash\{0\}} \frac{b(v, q)}{\|v\|_{\mathcal{X}}}+c(q, q) \geq \beta\|q\|_{\mathcal{M}} \text { for all } q \in \mathcal{M}
$$

is satisfied, then there is a unique pair $(u, p)$ in $\mathcal{V}=\mathcal{X} \times \mathcal{M}$ that solves (2.6).

REMARK 1. The boundedness and nonnegativity of $c$ ensures that the generalized saddle point problem (2.6) has a unique solution whenever (2.4) has a unique solution [10, Theorem 1].

REMARK 2. The saddle point problem (2.6) may have a solution even if $c$ is only weakly coercive [15].

The condition (2.9) is a standard second-order sufficient condition while the infsup condition (2.10) imposes restrictions on the constraints. In the standard saddle point system (2.4), $c \equiv 0$ and the inf-sup condition becomes

$$
\sup _{v \in \mathcal{X} \backslash\{0\}} \frac{b(v, q)}{\|v\|_{\mathcal{X}}} \geq \beta\|q\|_{\mathcal{M}} \text { for all } q \in \mathcal{M} .
$$

For the quadratic program (1.2), the inf-sup condition ensures that the constraint gradients are linearly independent since it is equivalent to

$$
\min _{q \in \mathbb{R}^{m} \backslash\{0\}} \max _{v \in \mathbb{R}^{n} \backslash\{0\}} \frac{q^{T} B v}{\|v\|_{2}\|q\|_{2}} \geq \beta,
$$

the left-hand side of which is a variational characterization of the smallest singular value of $B$. This shows that $B$ must have full rank, i.e., that the constraint gradients 
must be linearly independent, and guarantees that the minimizer $u$ of the quadratic program (1.2) is a regular point.

Thus, Theorem 2.1 shows that when $a$ is elliptic on $X$ and $c$ is nonnegative, whether the generalized saddle point system (2.6) has a unique solution depends on the inf-sup condition (2.10). This inf-sup condition is a general result that does not depend on the application; though it is well known in many continuous settings such as mixed finite element methods, it is equally applicable in discrete settings such as optimization where it is related to the linear independence of the constraint gradients.

Although Theorem 2.1 gives conditions for an inherently discrete problem to be solved numerically, in the next section we will see that when $\mathcal{X}$ and $\mathcal{M}$ are infinite dimensional a discrete analogue of the inf-sup condition is essential. Additionally, in later sections we will use the conditions on $a, b$ and $c$ described here, that are necessary for a unique solution to the original saddle point problem, to estimate the rate of convergence of certain preconditioned iterative methods for the saddle point system.

2.2. Discrete saddle point systems. In some applications, such as certain optimization or circuit problems, the saddle point system (2.6) involves finite dimensional Hilbert spaces $\mathcal{X}$ and $\mathcal{M}$ and the linear system (1.1) can be immediately obtained from (2.6). However, in other cases such as those involving partial differential equations, $\mathcal{X}$ and $\mathcal{M}$ may be infinite-dimensional, as we have already mentioned. If the infinite dimensional problem cannot be solved exactly, (2.6) is typically discretized in some fashion, perhaps by finite elements or radial basis functions, and a finite dimensional analogue of (2.4) is obtained. The discretization often depends on some parameter, such as the mesh width or the number of interpolation points, and we represent this dependence by a generic parameter $N$. We introduce families of finite dimensional subspaces $\left\{\mathcal{X}_{N}\right\}$ and $\left\{\mathcal{M}_{N}\right\}$ where $\mathcal{X}_{N} \subset \mathcal{X}$ and $\mathcal{M}_{N} \subset \mathcal{M}$ will generally depend on $N$. It is of course usually desirable that one has some approximability so that, for example, $\mathcal{X}_{N}$ becomes dense in $\mathcal{X}$ and $\mathcal{M}_{N}$ becomes dense in $\mathcal{M}$ as $N \rightarrow \infty$. Then an approximation of (2.6) is given by: find $\left(u_{N}, p_{N}\right) \in \mathcal{X}_{N} \times \mathcal{M}_{N}$ such that

$$
\begin{gathered}
a\left(u_{N}, v_{N}\right)+b\left(v_{N}, p_{N}\right)=f\left(v_{N}\right) \text { for all } v_{N} \in \mathcal{X}_{N} \\
b\left(u_{N}, q_{N}\right)-c\left(p_{N}, q_{N}\right)=g\left(q_{N}\right) \text { for all } q_{N} \in \mathcal{M}_{N} .
\end{gathered}
$$

The case that $\mathcal{X}$ and $\mathcal{M}$ are finite dimensional can be considered a special case of (2.12) in which $\mathcal{X}_{N}=\mathcal{X}$ and $\mathcal{M}_{N}=\mathcal{M}$ for all $N$.

A crucial issue arises when $c$ in the continuous saddle point problem (2.6) is not uniformly positive, since the inf-sup condition (2.10) in Theorem 2.1 may not be satisfied in the subspaces $\mathcal{X}_{N}$ and $\mathcal{M}_{N}$, even though it is satisfied in $\mathcal{X}$ and $\mathcal{M}$. In this case, a unique solution to the discrete problem does not necessarily exist. This can be remedied by stabilization, that is, by modifying $c$ or, in the case that $c \equiv 0$ in (2.6), by introducing a bounded, symmetric and nonnegative bilinear form $c$ on $\mathcal{M}_{N}$ (see, for example, Elman, Silvester and Wathen [27, Chapter 5], [25, Chapter 3] in the case of mixed finite element approximations of incompressible fluid dynamics problems).

Let us now focus in more detail on the conditions for a unique solution of the discrete system (2.12). Since $a$ is positive definite on $\mathcal{X}$, it is automatically positive definite on $\mathcal{X}_{N}$. Similarly, the nonnegativity of $c$ on $\mathcal{M}$ implies nonnegativity on $\mathcal{M}_{N}$. Thus, the only additional condition we are required to check is a discrete infsup condition [79, Theorem 2.6]. 
TheOREm 2.2. Let the conditions of Theorem 2.1 hold. If, additionally, there exists a constant $\beta>0$ for which

$$
\sup _{v_{N} \in \mathcal{X}_{N} \backslash\{0\}} \frac{b\left(v_{N}, q_{N}\right)}{\left\|v_{N}\right\|_{\mathcal{X}}}+c\left(q_{N}, q_{N}\right) \geq \beta\left\|q_{N}\right\|_{\mathcal{M}} \text { for all } q_{N} \in \mathcal{M}_{N}
$$

then there exists a unique solution $\left(u_{N}, p_{N}\right) \in \mathcal{V}_{N}=\mathcal{X}_{N} \times \mathcal{M}_{N}$ that solves (2.12).

REMARK 3. The inf-sup condition and its discrete counterpart play an important role in mixed methods for PDE problems, such as those arising in fluid dynamics [27, Chapter 5], [25, Chapter 3] and solid mechanics [11, Chapter VI]. However, researchers have begun to appreciate the importance of inf-sup conditions in other applications, such as when developing hybrid interpolants on the sphere [44, 64].

REMARK 4. As in Theorem 2.1, the inf-sup condition is related to the constraints and in the case $c \equiv 0$, it is equivalent to the condition that the constraint gradients are linearly independent.

Thus, if (2.6) is infinite dimensional, the way in which the finite dimensional spaces are chosen affects the additional discrete inf-sup condition (2.13) that must be satisfied. Certain choices may allow $c \equiv 0$ but for others it is necessary to include a stabilization term to ensure that (2.12) has a unique solution.

3. The matrix formulation. By selecting basis functions $\left\{\phi_{1}, \ldots \phi_{n}\right\}$ for $\mathcal{X}_{N}$ and $\left\{\psi_{1}, \ldots, \psi_{m}\right\}$ for $\mathcal{M}_{N}$, we can express the discrete generalized saddle point problem (2.12) in terms of the matrix equation (1.1) where

$$
A=\left[a_{i j}\right], a_{i j}=a\left(\phi_{i}, \phi_{j}\right), \quad B=\left[b_{k j}\right], b_{k j}=b\left(\phi_{j}, \psi_{k}\right), \quad C=\left[c_{k \ell}\right], \quad c_{i j}=c\left(\psi_{k}, \psi_{\ell}\right),
$$

$i, j=1, \ldots, n$ and $k, \ell=1, \ldots, m$. The conditions on the bilinear forms $a, b$ and $c$ given in Section 2 ensure that $A \in \mathbb{R}^{n \times n}$ is symmetric positive definite, $B \in \mathbb{R}^{m \times n}$, $m \leq n$ and $C \in \mathbb{R}^{m \times m}$ is symmetric positive semidefinite (and may be the zero matrix). However, $\mathcal{A}$ is always indefinite when $m>0$, with $n$ positive eigenvalues and $m$ negative eigenvalues [7, Section 3.4].

Once we choose bases we may represent the norms on $\mathcal{X}_{N}, \mathcal{M}_{N}$ and the product space $\mathcal{V}_{N}=\mathcal{X}_{N} \times \mathcal{M}_{N}$ using the Gram matrices $X, M$ and $V$. Specifically, we have that

$$
X=\left[x_{i j}\right], x_{i j}=\left(\phi_{i}, \phi_{j}\right)_{\mathcal{X}}, \quad M=\left[m_{k \ell}\right], m_{k \ell}=\left(\psi_{k}, \psi_{\ell}\right)_{\mathcal{M}}, \quad V=\left[\begin{array}{ll}
X & \\
& M
\end{array}\right],
$$

where, as previously mentioned, $(\cdot, \cdot)_{\mathcal{X}}$ and $(\cdot, \cdot)_{\mathcal{M}}$ are the inner products on the Hilbert spaces $\mathcal{X}$ and $\mathcal{M}$.

These Gram matrices allow us to develop equivalent conditions to those of Theorem 2.2 involving matrices $A, B$ and $C$ which will prove useful when considering preconditioners. In particular, the ellipticity and boundedness of $a$, from (2.1) and (2.9), and boundedness and nonnegativity of $c$, from (2.7) and (2.8), imply that for any nonzero $v \in \mathbb{R}^{n}$ and $q \in \mathbb{R}^{m}$,

$$
0<\alpha \leq \frac{v^{T} A v}{v^{T} X v} \leq \Gamma_{a} \text { for all } v \in \mathbb{R}^{n}
$$

and

$$
0 \leq \frac{q^{T} C q}{q^{T} M q} \leq \Gamma_{c} \text { for all } q \in \mathbb{R}^{m}
$$


For the inf-sup condition (2.13), we first note that for any $q \in \mathbb{R}^{m}$,

$$
\max _{\substack{v \in \mathbb{R}^{n} \\ v \neq 0}} \frac{q^{T} B v}{\left(v^{T} X v\right)^{\frac{1}{2}}}=\max _{\substack{w \in \mathbb{R}^{n} \\ w \neq 0}} \frac{q^{T} B X^{-\frac{1}{2}} w}{\left(w^{T} w\right)^{\frac{1}{2}}}=\left(q^{T} B X^{-1} B^{T} q\right)^{\frac{1}{2}},
$$

since the maximum is attained when $w$ is the unit vector in the direction of $X^{-\frac{1}{2}} B^{T} q$.

Accordingly, as a consequence of (2.13) we have that for any $q \in \mathbb{R}^{m}$

$$
\beta\left(q^{T} M q\right)^{\frac{1}{2}} \leq\left(q^{T} B X^{-1} B^{T} q\right)^{\frac{1}{2}}+\left(q^{T} C q\right)^{\frac{1}{2}} .
$$

Since, for any nonnegative numbers $d$ and $e,(\sqrt{d}+\sqrt{e})^{2} \leq 2(d+e)$, we find that

$$
\beta^{2} \leq 2 \frac{q^{T}\left(B X^{-1} B^{T}+C\right) q}{q^{T} M q} .
$$

The boundedness of $b$ (see (2.2)) also ensures that

$$
\Gamma_{b} \geq \max _{q \in \mathcal{M}_{N} \backslash\{0\}} \max _{v \in \mathcal{X}_{N} \backslash\{0\}} \frac{b(v, q)}{\|v\|_{\mathcal{X}}\|q\|_{\mathcal{M}}}
$$

or that

$$
\Gamma_{b} \geq \max _{q \in \mathbb{R}^{m} \neq 0} \frac{\left(q^{T} B X^{-1} B^{T} q\right)^{\frac{1}{2}}}{\left(q^{T} M q\right)^{\frac{1}{2}}} .
$$

Thus, for any $q \in \mathbb{R}^{m}$

$$
\frac{q^{T} B X^{-1} B^{T} q}{q^{T} M q} \leq \Gamma_{b}^{2} .
$$

The conditions (3.2), (3.3) and (3.6) are equivalent to conditions (2.1), (2.2), (2.7) and (2.9) in Theorem 2.2, while the inf-sup condition (3.5) differs from (2.13) only by a constant. Consequently, the discrete saddle point system (2.12) has a solution when (3.2)-(3.6) are satisfied.

Corollary 3.1. If positive constants $\Gamma_{a}, \Gamma_{b}, \Gamma_{c}, \alpha$ and $\beta$ exist such that (3.2)(3.6) are satisfied then there exists a unique vector $\left[u^{T}, p^{T}\right]^{T}$ that solves (1.1). Equivalently, the saddle point problem (2.12) has a unique solution in $\mathcal{X}_{N} \times \mathcal{M}_{N}$.

Corollary 3.1 shows that (3.2)-(3.6) are conditions for invertibility of the matrix $\mathcal{A}$. Note that although changing the bases for $\mathcal{X}_{N}$ and $\mathcal{M}_{N}$ will change the matrices $A, B$ and $C$, the constants $\Gamma_{a}, \Gamma_{b}, \Gamma_{c}, \alpha$ and $\beta$ are related to the underlying operators $a, b$ and $c$, and the spaces $\mathcal{X}_{N}$ and $\mathcal{M}_{N}$, and are independent of the bases chosen. They do, however, depend on the norms on the underlying spaces $\mathcal{X}_{N}$ and $\mathcal{M}_{N}$ and on the norms $\|\cdot\|_{\mathcal{X}}$ and $\|\cdot\|_{\mathcal{M}}$.

Of course, we can also determine the invertibility of $\mathcal{A}$ directly from the matrix. Since $A$ is invertible,

$$
\mathcal{A}=\left[\begin{array}{cc}
I & \\
B A^{-1} & I
\end{array}\right]\left[\begin{array}{cc}
A & \\
& -S
\end{array}\right]\left[\begin{array}{cc}
I & A^{-1} B^{T} \\
& I
\end{array}\right]
$$

where $S=B A^{-1} B^{T}+C$ is the (negative) Schur complement. Thus, $\mathcal{A}$ is invertible if and only if $S$ is invertible, which occurs if and only if $\operatorname{null}(C) \cap \operatorname{null}\left(B^{T}\right)=\{0\}[7$, 
Theorem 3.1]. Now, $\operatorname{null}(C) \cap \operatorname{null}\left(B^{T}\right)=\{0\}$ is equivalent to requiring that $B^{T} q$ and $C q$ are not simultaneously 0 for any $q \in \mathbb{R}^{m}, q \neq 0$, which is exactly the condition that must be satisfied if the right-hand side of (3.4) is to be nonzero. It follows that the condition $\operatorname{null}(C) \cap \operatorname{null}\left(B^{T}\right)=\{0\}$ is equivalent to finding some $\beta>0$ for which (3.4) is holds, and thus to satisfying the discrete inf-sup condition (2.13). This condition is automatically satisfied if $B$ has full rank or if $C$ is positive definite.

4. Solution by iterative methods. In the previous sections we examined conditions for a unique solution of the discrete standard and generalized saddle point systems to exist and showed that, by choosing basis functions for the finite dimensional spaces $\mathcal{X}_{N}$ and $\mathcal{M}_{N}$, we obtain the symmetric indefinite matrix system (1.1). For an ab initio discrete problem this certainly carries through with $\mathcal{X}_{N}=\mathcal{X}$ and $\mathcal{M}_{N}=\mathcal{M}$ for all $N$.

When $\mathcal{A}$ is large and sparse it is common to solve (1.1) by a Krylov subspace method. For saddle point problems MINRES is a popular solver for reasons that we elaborate on later in this section. Other methods, such as SYMMLQ [51], FOM [56], BiCGSTAB [67], QMR [30], TFQMR [29] and SQMR [31] may also be applied. However, the convergence of these methods is not so well understood and convergence bounds are in general difficult to obtain.

The rate of convergence of MINRES is usually heavily influenced by the spectrum of $\mathcal{A}$, although we note that the right-hand side can play a role. The convergence speed of other Krylov methods may also be affected by the eigenvalue distribution, although the connection between eigenvalues and convergence is usually less clear. In applications involving discretization, the speed of convergence often depends on the parameter $N$ and can deteriorate as the approximation to (2.6) becomes more accurate. In the ab initio discrete case, a slow rate of convergence may be caused by the spectral distribution.

The effect on the spectrum can be mitigated by using an appropriate preconditioner $\mathcal{P}$. Conceptually we can consider solving the equivalent linear system $\mathcal{P}^{-1} \mathcal{A} x=$ $\mathcal{P}^{-1} b$, although in practice we typically aim to preserve symmetry; this can certainly be achieved when $\mathcal{P}$ is symmetric positive definite. Ideally, the preconditioner should be chosen so that rate of convergence is independent of the parameter $N$ and it turns out that this is at least theoretically possible when certain block diagonal preconditioners of the form

$$
\mathcal{P}=\left[\begin{array}{ll}
P_{1} & \\
& P_{2}
\end{array}\right]
$$

are applied with $P_{1} \in \mathbb{R}^{n \times n}$ and $P_{2} \in \mathbb{R}^{m \times m}$ symmetric positive definite. The matrices $P_{1}^{-1}$ and $P_{2}^{-1}$ represent symmetric positive definite isomorphisms that map $\mathcal{X}_{N}^{\prime} \rightarrow \mathcal{X}_{N}$ and $\mathcal{M}_{N}^{\prime} \rightarrow \mathcal{M}_{N}$, where $\mathcal{X}_{N}^{\prime}$ is the dual space of $\mathcal{X}_{N}$ and $\mathcal{M}_{N}^{\prime}$ is the dual space of $\mathcal{M}_{N}[1,40,43,44,47,49,79]$. One choice, therefore, is $P_{1}=X$ and $P_{2}=M$, so that $\mathcal{P}=V$, with $V$ given by (3.1). By changing the spaces $\mathcal{X}_{N}$ or $\mathcal{M}_{N}$, or by altering the norm $V$, we may obtain more than one such preconditioner $\mathcal{P}$, and these need not be spectrally equivalent [47, Section 3$]$.

With the natural preconditioner $\mathcal{P}=V$ (see (3.1)), MINRES may be the natural Krylov subspace method to apply since it minimizes the residual with respect to the norm $\|\cdot\|_{V^{-1}}[1,37,61,71]$. For any $\left(u_{N}, p_{N}\right) \in \mathcal{X}_{N} \times \mathcal{M}_{N}$, the residual of the saddle point system (2.12) lies in the dual space $\mathcal{X}_{N}^{\prime} \times \mathcal{M}_{N}^{\prime}$ and, as a result, the Krylov subspace residual vector is associated with the norm on this space. In terms of the matrices in Section 3 this norm is $\|\cdot\|_{V^{-1}}$. That is, unlike in other Krylov methods, 
the norm that is minimized by MINRES is that associated with the underlying saddle point problem. A more detailed discussion of this point can be found in the article by Silvester and Simoncini [61] and in Elman, Silvester and Wathen [27, Chapter 6], [25, Chapter 4].

Many theoretically optimal preconditioners are too costly to apply in practice and must be replaced by more computationally feasible alternatives. It is possible to maintain $N$-independent convergence rates in this situation if the chosen $P_{1}$ and $P_{2}$ are spectrally equivalent to $X$ and $M$, i.e., if

$$
\delta \leq \frac{v^{T} P_{1} v}{v^{T} X v} \leq \Delta \text { for all } v \in \mathbb{R}^{n}
$$

and

$$
\theta \leq \frac{q^{T} P_{2} q}{q^{T} M q} \leq \Theta \text { for all } q \in \mathbb{R}^{m}
$$

for positive scalars $\delta, \Delta, \theta$ and $\Theta$. A discussion of spectrally equivalent preconditioners can be found in Section 10.1.3 of Benzi Golub and Liesen [7], with particular reference to problems involving elliptic partial differential equations and interior point methods. Mardal and Winther [47] also discuss multilevel approximations in the context of mixed finite elements.

As an example of a natural preconditioner, consider the Stokes problem from the introduction. Recall from Section 2.1 that in the infinite-dimensional variational problem, the relevant Hilbert spaces are $\mathcal{X}=H_{0}^{1}$ and $\mathcal{M}=L^{2}(\Omega)$. Thus, the saddle point operator maps $H_{0}^{1} \times L^{2}(\Omega)$ to the dual space $H_{0}^{-1} \times L^{2}(\Omega)$ and the corresponding optimal preconditioner (for this infinite-dimensional problem) is

$$
\mathcal{P}=\left[\begin{array}{cc}
\left(-\nabla^{2}\right) & 0 \\
0 & I
\end{array}\right]
$$

where $I$ is the identity on $L^{2}(\Omega)$, since $\mathcal{P}^{-1}$ maps $H_{0}^{-1} \times L^{2}(\Omega)$ onto $H_{0}^{1} \times L^{2}(\Omega)$. If $\mathcal{X}_{N} \subset \mathcal{X}$ and $\mathcal{M}_{N} \subset \mathcal{M}$ are chosen so that a stable finite element discretization is obtained then the preconditioner for the finite element approximation should be

$$
\mathcal{P}=\left[\begin{array}{cc}
A & 0 \\
0 & Q
\end{array}\right]
$$

where $A$ is the discrete stiffness matrix and $Q$ is the pressure mass matrix. In practice, $A$ is usually replaced by a spectrally equivalent operator, such as a fixed number of multigrid cycles, and $Q$ by its diagonal or by a fixed number of steps of Chebyshev semi-iteration [27, Chapter 6], [25, Chapter 4], [73].

Importantly, if bounds (4.2) and (4.3) can be obtained for $P_{1}$ and $P_{2}$ then these, in conjunction with (3.2)-(3.6), allow us to bound the eigenvalues of $\mathcal{P}^{-1} \mathcal{A}$ and determine how convergence rates of Krylov subspace methods may be affected by the preconditioner. For many Krylov methods convergence bounds are hard to derive, since the method does not minimize a suitable quantity such as the residual or error. The residual minimization property of MINRES means that we can obtain bounds on the convergence rate, yet even for this method determining precise bounds is difficult. (For more precise, but complicated, bounds for MINRES see Wathen, Fischer and Silvester [72].)

We quote just one convergence bound that shows the importance of eigenvalue bounds. Let the eigenvalues of $\mathcal{P}^{-1} \mathcal{A}$ be contained in the intervals $\left[-\mu_{m},-\mu_{1}\right] \cup\left[\nu_{1}, \nu_{n}\right]$ 
with $\mu_{m}-\mu_{1}=\nu_{n}-\nu_{1}$, so that the intervals are of equal length. Then after $2 k$ steps of MINRES the residual $r^{(2 k)}=b-\mathcal{A} x^{(2 k)}$ satisfies the bound [27, Theorem 6.13]

$$
\frac{\left\|r^{(2 k)}\right\|_{\mathcal{P}^{-1}}}{\left\|r^{(0)}\right\|_{\mathcal{P}^{-1}}} \leq 2\left(\frac{\sqrt{\mu_{m} \nu_{n}}-\sqrt{\mu_{1} \nu_{1}}}{\sqrt{\mu_{m} \nu_{n}}+\sqrt{\mu_{1} \nu_{1}}}\right)^{k} .
$$

The bound (4.5) can be pessimistic, particularly if the negative and positive eigenvalues of $\mathcal{P}^{-1} \mathcal{A}$ lie in intervals of significantly different lengths. However, it certainly shows that knowledge of the extreme eigenvalues of $\mathcal{P}^{-1} \mathcal{A}$ can provide useful information about the speed of convergence of MINRES. From the bound (4.5) we additionally discern that a sufficient condition for fast convergence is that $\mu_{m} / \mu_{1}$ and $\nu_{n} / \nu_{1}$ are small, since this will ensure that the eigenvalues are clustered away from the origin. The latter point is an important one since small eigenvalues can hinder the convergence rate of MINRES.

5. Bounds on the eigenvalues of the preconditioned matrix. Although several eigenvalue bounds for saddle point problems and preconditioners have been devised in various contexts, we now present general bounds for preconditioned saddle point problems with $A \succ 0$ that explicitly make use of the important boundedness, coercivity and inf-sup constants of the original saddle point formulation (2.6). Specifically, we bound the positive and negative eigenvalues of $\mathcal{P}^{-1} \mathcal{A}$ using the bounds (3.2)(3.6), that are related to the bilinear forms $a, b$ and $c$, and (4.2) and (4.3), that depend on the preconditioner $\mathcal{P}$.

Crucial to our proof of these bounds is Schur's determinant lemma [5, 57], that relates the determinant of a block matrix to the determinant of the Schur complement. The lemma itself is easily understood given the following decomposition of a block matrix

$$
\left[\begin{array}{cc}
E & F^{T} \\
F & -G
\end{array}\right]=\left[\begin{array}{cc}
I & 0 \\
F E^{-1} & I
\end{array}\right]\left[\begin{array}{cc}
E & 0 \\
0 & -G-F E^{-1} F^{T}
\end{array}\right]\left[\begin{array}{cc}
I & E^{-1} F^{T} \\
0 & I
\end{array}\right]
$$

which holds whenever $E$ is invertible. Since the determinant of a matrix product is the product of the determinants, we obtain Schur's result that

$$
\operatorname{det}\left(\left[\begin{array}{ll}
E & F^{T} \\
F & -G
\end{array}\right]\right)=\operatorname{det}(E) \operatorname{det}\left(-G-F E^{-1} F^{T}\right) .
$$

Analogously, if $G$ is invertible,

$$
\operatorname{det}\left(\left[\begin{array}{cc}
E & F^{T} \\
F & -G
\end{array}\right]\right)=\operatorname{det}(-G) \operatorname{det}\left(E+F^{T} G^{-1} F\right) .
$$

Another important component of the proof is the equivalence of the maxima of certain generalized Rayleigh quotients, which we show here.

LEMma 5.1. Let $B \in \mathbb{R}^{m \times n}, m \leq n$ have full rank and $X \in \mathbb{R}^{n \times n}, M \in \mathbb{R}^{m \times m}$ and $P_{2} \in \mathbb{R}^{m \times m}$ be symmetric positive definite. Then

$$
\max _{x \neq 0} \frac{x^{T} B^{T} P_{2}^{-1} B x}{x^{T} X x}=\max _{y \neq 0} \frac{y^{T} B X^{-1} B^{T} y}{y^{T} M y} \frac{y^{T} M y}{y^{T} P_{2} y} .
$$

Proof. By the Courant-Fischer theorem [39, Theorem 4.2.11],

$$
\max _{x \neq 0} \frac{x^{T} B^{T} P_{2}^{-1} B x}{x^{T} X x}=\max _{\widetilde{x} \neq 0} \frac{\widetilde{x}^{T} X^{-\frac{1}{2}} B^{T} P_{2}^{-1} B X^{-\frac{1}{2}} \widetilde{x}}{\widetilde{x}^{T} \widetilde{x}}=\lambda_{\max }
$$


where $\lambda_{\max }$ is the largest eigenvalue of $X^{-\frac{1}{2}} B^{T} P_{2}^{-1} B X^{-\frac{1}{2}} \in \mathbb{R}^{n \times n}$. Note that since $M$ and $P_{1}$ are positive definite and $B$ has rank $m$, the matrix $X^{-\frac{1}{2}} B^{T} P_{2}^{-1} B X^{-\frac{1}{2}}$ has $n-m$ zero eigenvalues and $m$ positive eigenvalues. Thus, $\lambda_{\max }>0$.

Since $X^{-\frac{1}{2}} B^{T} P_{2}^{-1} B X^{-\frac{1}{2}}$ and $X^{-1} B^{T} P_{2}^{-1} B$ are similar, $\lambda_{\max }$ is also the largest eigenvalue of $X^{-1} B^{T} P_{2}^{-1} B$ and there must be some nonzero vector $\hat{x} \in \mathbb{R}^{n}, \hat{x} \notin$ $\operatorname{null}(B)$ for which $X^{-1} B^{T} P_{2}^{-1} B \hat{x}=\lambda_{\max } \hat{x}$. Thus, $P_{2}^{-\frac{1}{2}} B X^{-1} B^{T} P_{2}^{-\frac{1}{2}} \hat{y}=\lambda_{\max } \hat{y}$, where $\hat{y}=P_{2}^{-\frac{1}{2}} B \hat{x}$ is nonsingular since $P_{2}$ is positive definite and $\hat{x} \notin \operatorname{null}(B)$. By again applying the Courant-Fischer theorem we obtain the result since

$$
\lambda_{\max }=\max _{\widetilde{y} \neq 0} \frac{\widetilde{y}^{T} P_{2}^{-\frac{1}{2}} B X^{-1} B^{T} P_{2}^{-\frac{1}{2}} \widetilde{y}}{\widetilde{y}^{T} \widetilde{y}}=\max _{y \neq 0} \frac{y^{T} B X^{-1} B^{T} y}{y^{T} M y} \frac{y^{T} M y}{y^{T} P_{2} y} .
$$

We are now in a position to state the bounds. Note that these bounds appeared previously in Silvester and Wathen [59] in the particular context of mixed finite elements for Stokes equations. The theorem below is slightly more general, since we do not assume that $X=A$ and $M=Q$, but the result is essentially the same.

TheOREM 5.2. Let $\mathcal{A}$ in (1.1) be preconditioned by $\mathcal{P}$ in (4.1) and let $\Gamma_{a}$ in (2.1) and (3.2) be greater than or equal to one. Then, negative eigenvalues $\lambda$ of the preconditioned matrix $\mathcal{P}^{-1} \mathcal{A}$ satisfy

$$
\lambda \in\left[-\theta^{-1}\left(\Gamma_{c}+\frac{\Gamma_{b}^{2}}{\alpha}\right), \frac{1}{2}\left(\frac{\Gamma_{a}}{\Delta}-\sqrt{\left(\frac{\Gamma_{a}}{\Delta}\right)^{2}+\frac{2 \beta^{2}}{\Theta \Delta}}\right)\right]
$$

while positive eigenvalues $\lambda$ satisfy

$$
\lambda \in\left[\frac{\alpha}{\Delta}, \frac{1}{2}\left(\frac{\Gamma_{a}}{\delta}+\sqrt{\left(\frac{\Gamma_{a}}{\delta}\right)^{2}+\frac{4 \Gamma_{b}^{2}}{\theta \delta}}\right)\right] .
$$

Proof. Any eigenvalue $\lambda$ of $\mathcal{P}^{-1} \mathcal{A}$ is an eigenvalue of the generalized problem $\mathcal{A} w=\lambda \mathcal{P} w$ for some nonzero vector $w \in \mathbb{R}^{n+m}$. Equivalently, $\mathcal{A}-\lambda \mathcal{P}$ must be singular, so that

$$
\operatorname{det}\left(\left[\begin{array}{cc}
A-\lambda P_{1} & B^{T} \\
B & -\left(C+\lambda P_{2}\right)
\end{array}\right]\right)=0
$$

Let us consider the cases $\lambda>0$ and $\lambda<0$ separately. If $\lambda>0$, then $C+\lambda P_{2}$ is symmetric positive definite and by Schur's determinant lemma, (5.1) holds if and only if $\operatorname{det}\left(\left(A-\lambda P_{1}\right)+B^{T}\left(C+\lambda P_{2}\right)^{-1} B\right)=0$. The determinant of a matrix is the product of its eigenvalues and so

$\lambda_{\min }\left(\left(A-\lambda P_{1}\right)+B^{T}\left(C+\lambda P_{2}\right)^{-1} B\right) \leq 0 \leq \lambda_{\max }\left(\left(A-\lambda P_{1}\right)+B^{T}\left(C+\lambda P_{2}\right)^{-1} B\right)$.

Since $B \in \mathbb{R}^{m \times n}(m \leq n)$ has rank at most $m$, the $n \times n$ positive semidefinite matrix $B^{T}\left(C+\lambda P_{2}\right)^{-1} B$ also has rank at most $m$ and $\lambda_{\min }\left(B^{T}\left(C+\lambda P_{2}\right)^{-1} B\right)=0$. Combining this result with Weyl's inequality [39, Theorem 4.3.1] and (5.2) shows that

$$
\lambda_{\min }\left(A-\lambda P_{1}\right) \leq 0 \leq \lambda_{\max }\left(A-\lambda P_{1}\right)+\lambda_{\max }\left(B^{T}\left(C+\lambda P_{2}\right)^{-1} B\right) .
$$


From the lower bound in (5.3) and the Courant-Fischer theorem [39, Theorem 4.2.11] we find that

$$
0 \geq \lambda_{\min }\left(A-\lambda P_{1}\right)=\min _{x \neq 0} \frac{x^{T}\left(A-\lambda P_{1}\right) x}{x^{T} x}
$$

so that

$$
\min _{x \neq 0} \frac{x^{T} A x}{x^{T} X x} \leq \lambda \max _{x \neq 0} \frac{x^{T} P_{1} x}{x^{T} X x} .
$$

Thus, using (3.2) and (4.2), we obtain the lower bound for positive eigenvalues.

We now turn to the upper bound in (5.3). Since $\left(\lambda P_{2}\right)^{-1} \succeq\left(C+\lambda P_{2}\right)^{-1}$,

$0 \leq \lambda_{\max }\left(A-\lambda P_{1}\right)+\frac{1}{\lambda} \lambda_{\max }\left(B^{T} P_{2}^{-1} B\right)=\max _{x \neq 0} \frac{x^{T}\left(A-\lambda P_{1}\right) x}{x^{T} x}+\frac{1}{\lambda} \max _{x \neq 0} \frac{x^{T} B^{T} P_{2}^{-1} B x}{x^{T} x}$,

where again we have used the Courant-Fischer theorem. It follows from (3.2), (3.6), Lemma 5.1 and (4.2) that

$$
0 \leq \Gamma_{a}-\delta \lambda+\frac{1}{\lambda} \frac{\Gamma_{b}^{2}}{\theta}
$$

Consequently, $\lambda^{2}-\left(\Gamma_{a} / \delta\right) \lambda-\Gamma_{b}^{2} /(\theta \delta) \leq 0$, the solution of which gives the upper bound.

Let us now consider $\lambda<0$. With this choice, $A-\lambda P_{1}$ is symmetric positive definite and (5.1) is equivalent to $\operatorname{det}\left(\left(C+\lambda P_{2}\right)+B\left(A-\lambda P_{1}\right)^{-1} B^{T}\right)=0$ by Schur's determinant lemma. Again we infer that

$$
\lambda_{\min }\left(\left(C+\lambda P_{2}\right)+B\left(A-\lambda P_{1}\right)^{-1} B^{T}\right) \leq 0 \leq \lambda_{\max }\left(\left(C+\lambda P_{2}\right)+B\left(A-\lambda P_{1}\right)^{-1} B^{T}\right)
$$

so applying Weyl's inequality gives $\lambda_{\min }\left(C+\lambda P_{2}\right)+\lambda_{\min }\left(B\left(A-\lambda P_{1}\right)^{-1} B^{T}\right) \leq 0$ and $0 \leq \lambda_{\max }\left(C+\lambda P_{2}\right)+\lambda_{\max }\left(B\left(A-\lambda P_{1}\right)^{-1} B^{T}\right)$. Since $A^{-1} \succeq\left(A-\lambda P_{1}\right)^{-1}$, the upper bound, in conjunction with $(3.3),(4.3),(3.2)$ and (3.6), gives

$$
0 \leq \max _{y \neq 0} \frac{y^{T}\left(C+\lambda P_{2}\right) y}{y^{T} y}+\max _{y \neq 0} \frac{y^{T} B A^{-1} B^{T} y}{y^{T} y} \leq \Gamma_{c}+\theta \lambda+\frac{\Gamma_{b}^{2}}{\alpha} .
$$

Thus, $\lambda \geq-\theta^{-1}\left(\Gamma_{c}+\Gamma_{b}^{2} / \alpha\right)$, which is the lower bound for negative eigenvalues.

For the upper bound on negative eigenvalues, we find that

$$
0 \geq \min _{y \neq 0} \frac{y^{T}\left(C+\lambda P_{2}\right) y}{y^{T} y}+\min _{y \neq 0} \frac{y^{T} B\left(A-\lambda P_{1}\right)^{-1} B^{T} y}{y^{T} y} .
$$

From (3.2) and (4.2) we find that, for any $x \in \mathbb{R}^{n}, x^{T}(A-\lambda P) x \leq\left(\Gamma_{a}-\lambda \Delta\right) x^{T} X x$ and so

$$
\begin{aligned}
0 & \geq \min _{y \neq 0} y^{T}\left(\lambda P_{2}+C\right) y+\frac{1}{\Gamma_{a}-\lambda \Delta} \min _{y \neq 0} y^{T} B X^{-1} B^{T} y \\
& \geq \min _{y \neq 0} \lambda y^{T} P_{2} y+\frac{1}{\Gamma_{a}-\lambda \Delta} \min _{y \neq 0} y^{T}\left(B X^{-1} B^{T}+C\right) y,
\end{aligned}
$$

using the assumption that $\Gamma_{a} \geq 1$. From (3.5) and (4.3) we obtain $\lambda \Theta+\beta^{2} / 2\left(\Gamma_{a}-\right.$ $\lambda \Delta) \leq 0$, the solution of which gives the upper bound on negative eigenvalues. 
Remark 5. As has been shown previously [27, Theorem 6.6] the upper bound on positive eigenvalues can be made simpler, although less sharp, by completing the square of the term under the square root sign. Doing so gives the bound for positive eigenvalues $\lambda \in\left[\alpha / \Delta, \Gamma_{a} / \delta+\Gamma_{b}^{2} /\left(\theta \Gamma_{a}\right)\right]$.

Using (4.5), the bounds of Theorem 5.2 can be used to infer information about the speed of convergence of MINRES applied to $\mathcal{P}^{-1} \mathcal{A}$. We note that even for direct methods these bounds have implications, since the accuracy of the computed solution can be poor if $\mathcal{A}$ is ill-conditioned. Theorem 5.2 shows that the condition number (of the unpreconditioned problem) depends on the constants $\alpha, \beta, \Gamma_{a}, \Gamma_{b}$ and $\Gamma_{c}$ and that the sizes, and not just the existence, of the coercivity constant $\alpha$ and inf-sup constant $\beta$ may be important when solving saddle point systems by direct or iterative methods.

When $\delta=\Delta=\theta=\Theta=1$ we have the theoretically optimal preconditioner defined in the previous section and the eigenvalue bounds in Theorem 5.2 reduce to

$$
\lambda \in\left[-\left(\Gamma_{c}+\frac{\Gamma_{b}^{2}}{\alpha}\right), \frac{1}{2}\left(\Gamma_{a}-\sqrt{\Gamma_{a}^{2}+2 \beta^{2}}\right)\right] \cup\left[\alpha, \frac{1}{2}\left(\Gamma_{a}+\sqrt{\Gamma_{a}^{2}+4 \Gamma_{b}^{2}}\right)\right] .
$$

Ensuring that the eigenvalues of $\mathcal{P}^{-1} \mathcal{A}$ are clustered away from the origin in this case reduces to ensuring that $\alpha$, the coercivity constant for $a$, and $\beta$, the inf-sup constant, are not too small, and that the boundedness constants $\Gamma_{a}, \Gamma_{b}$ and $\Gamma_{c}$ are not too large. Recall that these constants are independent of the choice of bases but do depend on the spaces $\mathcal{X}_{N}$ and $\mathcal{M}_{N}$, an on the norms on these spaces. As the ratios $\Delta / \delta$ and $\Theta / \theta$ get larger, so too do the intervals in Theorem 5.2 that contain the eigenvalues of $\mathcal{P}^{-1} \mathcal{A}$. Since $\Delta / \delta$ and $\Theta / \theta$ determine how well $P_{1}$ and $P_{2}$ approximate $X$ and $M$, it is desirable to have preconditioners that approximate these Gram matrices well.

6. Applications of Theorem 5.2. In this section we examine the results of previous sections in the context of the Stokes flow, hybrid interpolation and circuit problems introduced in Section 1.

6.1. Stokes flow. We begin with the Stokes equations, which have appeared throughout this manuscript. We discretize the variational equations (2.5) by $Q_{1}-Q_{1}$ finite elements, although similar results hold for other stabilized elements and for stable elements. By choosing appropriate basis functions we obtain a saddle point system (1.1) with Gram matrices $X=A$ and $M=Q$, the pressure mass matrix, since $\mathcal{X}=H_{0}^{1}$ and $\mathcal{M}=L_{2}(\Omega)$. If we assume that the stabilization matrix satisfies the ideal stabilization property $q^{T} C q / q^{T} Q q \leq 1$ for all $p \in \mathbb{R}^{m}[27$, page 276], the finite dimensional generalized saddle point problem satisfies the conditions of Theorem 2.2. In this case, on a shape regular, quasi-uniform subdivision of $\mathbb{R}^{2}$, the constants in (3.2)-(3.6) are $\alpha=\Gamma_{a}=1, \Gamma_{c}=1, \Gamma_{b}^{2}=3$ and the inf-sup constant $\beta$ is bounded away from zero independently of the mesh parameter $h$ [27, Theorem 5.28]. On square grids on $[-1,1]^{2}$, numerical evidence suggests that $\beta^{2}>2 / 5[27$, page 277$]$.

The preconditioner (4.4) is theoretically optimal but too costly to apply and so we replace $A$ by a multigrid $\mathrm{V}$-cycle and $Q$ by its diagonal. Computation of the eigenvalues of $P_{1}^{-1} A$ using IFISS [26, 58] suggests that the constants in (4.2) are $\delta=1$ and $\Delta=1.25$, while it is known that $\theta=1 / 2$ and $\Theta=3 / 2$ in (4.3) [75]. Thus, letting $\beta^{2}=2 / 5$, Theorem 5.2 guarantees that the eigenvalues of $\mathcal{P}^{-1} \mathcal{A}$ lie in $[-8,-0.11] \cup[0.8,3]$. For comparison, we discretize the channel problem in Elman, Silvester and Wathen [27, Section 5.1.1], which is defined by the analytic solution $u=$ $\left(1-y^{2}, 0\right)$ on $[-1,1]^{2}$ with a natural outflow condition, using a mesh width of $2^{-5}$, and 
compute the eigenvalues of $\mathcal{P}^{-1} \mathcal{A}$ for the preconditioner described. These eigenvalues lie in $[-1.34,-0.25] \cup[0.80,2.24]$, which shows that the bounds in Theorem 5.2 are reasonable. Note that the lower bound on positive eigenvalues is particularly close. Numerical experiments show that the preconditioner gives mesh-independent iteration counts and is very effective [27, Table 6.1].

6.2. Hybrid interpolation. For the hybrid interpolation method, we consider interpolation at a set of points on the sphere $X_{N}=\left\{x_{j}\right\}_{j=1, \ldots, N}$. Assuming that the $N$ data points are distinct, we can construct a radial basis approximation space $\mathcal{X}_{N}$ with associated native space norm (see, for example, Le Gia, Sloan and Wathen [44] for more details) and a spherical polynomial approximation space $\mathcal{M}_{L}$, also equipped with the native space norm, where $L \geq 0$ is the maximum total degree of the polynomials. The interpolant is $u_{N, L}\left(\boldsymbol{x}_{i}\right)+p_{N, L}\left(\boldsymbol{x}_{i}\right)=f\left(\boldsymbol{x}_{i}\right)$, where

$$
u_{N, L}(\boldsymbol{x})=\sum_{j=1}^{N} \alpha_{j} \phi\left(\boldsymbol{x}, \boldsymbol{x}_{j}\right) \in \mathcal{X}_{N}
$$

is the radial basis function interpolant with strictly positive definite and radially symmetric kernel function $\phi$ and

$$
p_{N, L}=\sum_{\ell=0}^{L} \sum_{k=0}^{M(d, \ell)} \beta_{\ell, k} Y_{\ell, k}(\boldsymbol{x}) \in \mathcal{M}_{L}
$$

is the spherical harmonic interpolant with spherical harmonic functions $Y_{\ell, k}$. Here, $M(d, \ell)$ is the dimension of the space spanned by the spherical harmonics of degree $\ell$. For uniqueness of the interpolant we require that $u_{N, L}$ is orthogonal to all $q \in \mathcal{M}_{L}$, with respect to the native space norm.

The bilinear forms $a$ and $b$ are both defined by the native space inner product. Since this is also the norm on $\mathcal{X}_{N}, \alpha=\Gamma_{a}=1$. Additionally, $\Gamma_{b}=1$ [44, page 705]. When the mesh norm

$$
h_{X}=\sup _{x \in \mathbb{S}^{d}} \inf _{x_{j} \in X_{N}} \cos ^{-1}\left(x \cdot x_{j}\right)
$$

satisfies $h_{X} \leq \tau / L$, where $\tau>0$ is a specified constant [64], the inf-sup condition (2.13) is satisfied, with inf-sup constant $\beta$ that is independent of $N$ but which depends on $L$, and Theorem 2.2 holds [44, 64, Theorem 2]. From experiments it appears that when $N=4000$ then $\beta$ is no smaller than 0.8 for $m=0,1, L=5,10,15,20,25$. Note that for fixed point set $X_{N}$ but increasing $L$ the inf-sup condition eventually breaks down.

To recover the coefficients $\alpha_{j}$ and $\beta_{\ell, k}$ we must solve a saddle point system (1.1) with $A_{i j}=\phi\left(x_{i}, x_{j}\right), i, j=1, \ldots, N$, and $B_{\ell k, i}=Y_{k, \ell}\left(x_{i}\right), i=1, \ldots, N, k=$ $1, \ldots, M(d, \ell)$ and $\ell=0, \ldots, L$. Thus, $A \in \mathbb{R}^{N \times N}, B \in \mathbb{R}^{N \times M}$ and $C=0$, where

$$
M=\sum_{\ell=0}^{L} M(d, \ell)=\operatorname{dim}\left(\mathcal{M}_{L}\right) .
$$

An effective natural preconditioner (4.1) was devised by Le Gia, Sloan and Wathen [44] which we now describe. Since $a$ is equivalent to the norm on $\mathcal{X}_{N}$, the preconditioner $P_{1}$ should be spectrally equivalent matrix to $A$ and Le Gia, Sloan and 
Wathen use a domain decomposition preconditioner. Examination of the inf-sup condition shows that the right choice for $P_{2}$ is a diagonal matrix which is also extremely efficient to apply. Knowledge of $\delta$ and $\Delta$ would allow us to compute the bounds in Theorem 5.2, which guarantee $N$-independent convergence. Certainly, for fixed $L$, the number of preconditioned MINRES iterations remains approximately constant with $N$ [44, Table 2].

6.3. Circuit. Current in a connected resistive network is modelled by Kirchoff's voltage law, which states that voltage drops in a loop add to zero and is associated with $B^{T}$, Kirchoff's current law, which states that currents at nodes add to zero and is associated with $B$, and Ohm's law, which links voltages and currents across resistors and is associated with $A$. The matrix $A$ is a diagonal matrix of inverse resistances of resistors in the circuit while $B^{T}$ is an incidence matrix [65].

In a connected circuit, $B^{T}$ does not have full rank since the vector of all ones is in its nullspace. A physical interpretation in terms of voltages is that if the voltages at each node are the same, the potential differences are all zero. The Schur complement $B A^{-1} B^{T}$ produced by this rank-deficient $B^{T}$ is a semidefinite weighted Laplacian and is called the conductance matrix. Its second-smallest eigenvalue $\lambda_{2}$ is called the algebraic connectivity [28] and is positive, since the circuit is connected. The eigenvalue $\lambda_{2}$ is important in graph theory [48] and its size is a measure of the connectedness of the circuit.

By grounding one node in the circuit we remove one degree of freedom. The reduced matrix $B^{T}$ is full rank and we can determine the potentials of nodes relative to that of the grounded node. The grounded network gives a symmetric positive definite Schur complement and its smallest eigenvalue, $\beta$ in the inf-sup condition (2.13), is a lower bound on the algebraic connectivity. It can also be related to the path resistance, or effective resistance [24]. We find that $A$ is easy to precondition since it is a diagonal matrix, while the Schur complement, is a grounded or Dirichlet weighted Laplacian, and preconditioners for such matrices, such as multigrid, could be used.

7. Results for semidefinite $A$. To this point we have assumed that $A$ is positive definite, i.e., that the bilinear form $a$ is coercive on the whole space $\mathcal{X}$. However, in many applications, $A$ is semidefinite but is positive definite on the nullspace of $B$, i.e., $a$ is coercive on a subspace of $\mathcal{X}$ that we define below. In this section we outline similar results to those in Sections $2-5$ for these types of problems.

To define an analogous saddle point system to (2.6) we first introduce the subspace $\mathcal{W} \subset \mathcal{X}$, where $\mathcal{W}=\{v \in \mathcal{X}: b(v, q)=0$ for all $q \in \mathcal{M}\}$ is the nullspace of $b$. Then the generalization of (2.6) we consider is that $a$ is symmetric and nonnegative and is coercive on $\mathcal{W}$, i.e., there exists a positive constant $\alpha_{\mathcal{W}}$ such that $a(v, v) \geq \alpha_{\mathcal{W}}\|v\|_{\mathcal{X}}^{2}$ for all $v \in \mathcal{W}$. The other conditions on $a, b$ and $c$, namely, (2.1), (2.2) and (2.7) still hold.

Then, the generalized saddle point problem (2.6) has a unique solution provided that the inf-sup condition (2.13), i.e., the inf-sup condition with $c$ removed, holds [11, Chapter III, Lemma 4.23]. Analogously to Theorem 2.2, if we want a unique solution to the discrete saddle point problem (2.12) we must satisfy the coercivity and infsup conditions on the finite-dimensional spaces $\mathcal{X}_{N}, \mathcal{M}_{N}$ and $\mathcal{W}_{N}$. An important point is that if $\mathcal{W}_{N} \not \subset \mathcal{W}$ we must ensure that $a$ is coercive on $\mathcal{W}_{N}$. By choosing basis vectors, as in Section 3, we arrive at the saddle point system (1.1) but with $A$ symmetric positive semi-definite and positive definite on the nullspace of $B$. The extensions of Theorems 2.1 and 2.2 ensure that the saddle point matrix $\mathcal{A}$ is invertible and has $n$ positive eigenvalues and $m$ negative eigenvalues $[19,35]$. Natural symmetric 
block diagonal preconditioners (4.1) that are determined by the spaces $\mathcal{X}$ and $\mathcal{M}$ are still appropriate and the resulting preconditioned system can be solved by the same preconditioned iterative methods as for the case that $A$ is positive definite.

The eigenvalues of $\mathcal{P}^{-1} \mathcal{A}$ are still relevant to estimating the rate of convergence of Krylov solvers since $\mathcal{A}$ is symmetric $\mathcal{P}$ symmetric positive definite. Bounds of the type in Theorem 5.2 were obtained by Gould and Simoncini [36] and although these are given for unpreconditioned matrices they can be applied to the transformed saddle point matrix $\mathcal{P}^{-\frac{1}{2}} \mathcal{A} \mathcal{P}^{-\frac{1}{2}}$. When $C=0$, the eigenvalue bounds are similar to those for the case that $A$ is positive definite (although the smallest value of $x^{T} A x / x^{T} X x$ may be negative), with the exception of the lower bound on positive eigenvalues. To apply this lower bound we must additionally compute the smallest eigenvalue $\mu$ of $A$ restricted to the nullspace of $B$, or a lower bound on $\mu$, and find the smallest root of a cubic equation. In the case of nonzero $C$, application of the bounds depends on the smallest eigenvalue of $A$ on the nullspace of $B$ being large enough.

8. Conclusions. In this manuscript we have described the necessary components for solving standard and generalized saddle point problems by iterative methods, irrespective of the application. In particular, we have given conditions for the unique solution of the saddle point problem, and shown that a sufficient ingredient is fulfilment of an inf-sup condition. When the saddle point problem requires discretization, another inf-sup condition must be satisfied on the finite dimensional discretization space.

To numerically solve the saddle point system we must solve a linear system involving a saddle point matrix, and conditions for its invertibility follow straightforwardly from those of the saddle point problem. Moreover, the spaces on which the saddle point problem are posed provide guidance for choosing a suitable block diagonal preconditioner. We have given bounds on the eigenvalues of the block preconditioned saddle point matrix and have indicated how they can be used to estimate the convergence rate of the MINRES Krylov subspace method applied to the linear system. These bounds show that coercivity and boundedness constants, as well as the infsup constant, are not only important for determining when the saddle point problem can be solved. These constants are also integral to the whole solution process and, in conjunction with suitable bounds on the preconditioner blocks, provide valuable information about the solution of the linear system by preconditioned iterative methods.

Acknowledgements We gratefully acknowledge the anonymous referees for their pertinent and perceptive comments which have significantly improved our exposition. This publication was based on work supported in part by Award No KUK-C1-013-04, made by King Abdullah University of Science and Technology (KAUST).

\section{REFERENCES}

[1] M. Arioli And D. Loghin, Stopping criteria for mixed finite element problems, Electron. Trans. Numer. Anal., 29 (2008), pp. 178-192.

[2] D. N. Arnold, R. S. Falk, And R. Winther, Preconditioning discrete approximations of the Reissner-Mindlin plate model, RAIRO Modél. Math. Anal. Numér., 31 (1997), pp. 517557.

[3] O. Axelsson and M. Neytcheva, Eigenvalue estimates for preconditioned saddle point matrices, Numer. Linear Algebra Appl., 13 (2006), pp. 339-360.

[4] I. BABUŠKA, Error-bounds for finite element method, Numer. Math., 16 (1971), pp. 322-333. 
[5] T. Banachiewicz, Zur Berechnung der Determinanten, wie auch der Inversen, und zur darauf basierten Auflösung der Systeme linearen Gleichungen, Acta Astronom. Ser. C, 3 (1937), pp. 41-67.

[6] M. Benzi And G. H. Golub, A preconditioner for generalized saddle point problems, SIAM J. Matrix Anal. Appl., 26 (2004), pp. 20-41.

[7] M. Benzi, G. H. Golub, AND J. LiEsen, Numerical solution of saddle point problems, Acta Numer., 14 (2005), pp. 1-137.

[8] M. Benzi And M. K. NG, Preconditioned iterative methods for weighted Toeplitz least squares problems, SIAM J. Matrix Anal. Appl., 27 (2006), pp. 1106-1124.

[9] P. B. Bochev AND R. B. LehoucQ, Regularization and stabilization of discrete saddle-point variational problems, Electron. Trans. Numer. Anal., 22 (2006), pp. 97-113.

[10] D. Braess, Stability of saddle point problems with penalty, RAIRO Modél. Math. Anal. Numér., 30 (1996), pp. 731-742.

[11] _ Finite elements: Theory, fast solvers, and applications in solid mechanics, Cambridge University Press, Cambridge, UK, 2007.

[12] J. H. Bramble and J. E. Pasciak, A preconditioning technique for indefinite systems resulting from mixed approximations of elliptic problems, Math. Comp., 50 (1988), pp. 1-17.

[13] J. H. Bramble AND J. E. PASCiAK, Iterative techniques for time dependent Stokes problems, Comput. Math. Appl., 33 (1997), pp. 13-30.

[14] F. BREZZI, On the existence, uniqueness and approximation of saddle-point problems arising from Lagrangian multipliers, RAIRO Anal. Numér., 8 (1974), pp. 129-151.

[15] F. Brezzi And M. Fortin, Mixed and Hybrid Finite Element Methods, Springer-Verlag, 1991.

[16] B. M. Brown, P. K. Jimack, And M. D. Minajlović, An efficient direct solver for a class of mixed finite element problems, Appl. Numer. Math., 38 (2001), pp. 1-20.

[17] R. H. Byrd, F. E. Curtis, And J. Nocedal, An inexact Newton method for nonconvex equality constrained optimization, Math. Program., Ser. A, 122 (2010), pp. 273-299.

[18] J. Cahouet And J.-P. Chabard, Some fast 3D finite element solvers for the generalized Stokes problem, Internat. J. Numer. Methods Fluids, 8 (1988), pp. 869-895.

[19] Y. Chabrillac and J.-P. Crouzeix, Definiteness and semidefiniteness of quadratic forms revisited, Linear Algebra Appl., 63 (1984), pp. 283-292.

[20] H.-H. Chen and J. C. Strikwerda, Preconditioning for regular elliptic systems, SIAM J. Numer. Anal., 37 (1999), pp. 131-151.

[21] Z. Chen, R. E. Ewing, AND R. LAZARov, Domain decomposition algorithms for mixed methods for second-order elliptic problems, Math. Comp, 65 (1996), pp. 467-490.

[22] H. S. Dollar, N. I. M. Gould, W. H. A. Schilders, ANd A. J. Wathen, Implicit-factorization preconditoning and iterative solvers for regularized saddle-point systems, SIAM J. Matrix Anal. Appl., 28 (2006), pp. 170-189.

[23] H. S. Dollar, N. I. M. Gould, and A. J. Wathen, On implicit-factorization constraint preconditioners, in Large-Scale Nonlinear Optimization, G. Di Pillo and M. Roma, eds., Springer Verlag, 2006, pp. 61-82.

[24] W. Ellens, F. M. Spieksma, P. Van Mieghem, A. Jamakovic, and R. E. Kooij, Effective graph resistance, Linear Algebra Appl., 435 (2011), pp. 2491-2506.

[25] H. Elman, D. Silvester, And A. Wathen, Finite Elements and Fast Iterative Solvers: with applications in incompressible fluid dynamics, Oxford University Press, Oxford, second ed., 2014.

[26] H. C. Elman, A. Ramage, and D. J. Silvester, Algorithm 866: IFISS, a Matlab toolbox for modelling incompressible flow, ACM Trans. Math. Software, 33 (2007), pp. 1-14.

[27] H. C. Elman, D. J. Silvester, And A. J. Wathen, Finite Elements and Fast Iterative Solvers: with applications in incompressible fluid dynamics, Oxford University Press, Oxford, 2005.

[28] M. FIEDLER, A property of eigenvectors of nonnegative symmetric matrices and its application to graph theory, Czechoslovak Mathematical Journal, 25 (1975), pp. 619-633.

[29] R. W. Freund, A transpose-free quasi-minimal residual algorithm for non-Hermitian linear systems, SIAM J. Sci. Comput., 14 (1993), pp. 470-482.

[30] R. W. Freund and N. M. Nachtigal, QMR: A quasi-minimal residual method for nonHermitian linear systems, Numer. Math., 60 (1991), pp. 315-339.

[31] R. W. Freund And H. Zha, Simplfications of the nonsymmetric Lanczos process and a new algoithm for Hermitian indefinite linear systems, tech. report, AT\&T Bell Labs, 1994.

[32] R. Glowinski and O. Pironneau, Numerical methods for the first biharmonic equation and for the two-dimensional Stokes problem, SIAM Rev., 21 (1979), pp. 167-212.

[33] R. Glowinski And M. F. Wheeler, Domain decomposition and mixed finite element methods for elliptic problems, in First International Symposium on Domain Decomposition Methods for Partial Differential Equations, R. Glowinski, G. Golub, G. Meurant, and J. Periaux, 
eds., SIAM, Philadelphia, PA, 1988, pp. 144-172.

[34] G. H. Golub AND C. GReIF, On solving block-structured indefinite linear systems, SIAM J. Sci. Comput., 24 (2003), pp. 2076-2092.

[35] N. I. M. Gould, On practical conditions for the existence and uniqueness of solutions to the general equality quadratic programming problem, Math. Program., 32 (1985), pp. 90-99.

[36] N. I. M. Gould And V. Simoncini, Spectral analysis of saddle point matrices with indefinite leading blocks spectral analysis of saddle point matrices with indefinite leading blocks, SIAM J. Matrix Anal. Appl., 31 (2009), pp. 1152-1171.

[37] A. Günnel and R. Herzog an E. SaAKs, A note on preconditioners and scalar products in Krylov subspace methods for self-adjoint problems in Hilbert space, Electron. Trans. Numer. Anal., 41 (2014), pp. 13-20.

[38] R. Hiptmair, Operator preconditioning, Comput. Math. Appl., 52 (2006), pp. 699-706.

[39] R. A. Horn and C. R. Johnson, Matrix Analysis, Cambridge University Press, New York, NY, 1990.

[40] I. C. F. IPSEn, A note on preconditioning nonsymmetric matrices, SIAM J. Sci. Comput., 23 (2001), pp. 1050-1051.

[41] C. Keller, N. I. M. Gould, And A. J. Wathen, Constraint preconditioning for indefinite linear systems, SIAM J. Matrix Anal. Appl., 21 (2000), pp. 1300-1317.

[42] A. Klawonn, Block-triangular preconditioners for saddle point problems with a penalty term, SIAM J. Sci. Comput., 19 (1998), pp. 172-184.

[43] Yu. A. KuzNetsov, Efficient iterative solvers for elliptic finite element problems on nonmatching grids, Russ. J. Numer. Anal. Math. Modelling, 10 (1995), pp. 187-211.

[44] Q. T. Le Gia, I. H. Slonn, and A. J. Wathen, Stability and preconditioning for a hybrid approximation on the sphere, Numer. Math., 118 (2011), pp. 695-711.

[45] D. Loghin And A. J. Wathen, Analysis of preconditioners for saddle-point problems, SIAM J. Sci. Comput., 25 (2004), pp. 2029-2049.

[46] L. LuKŠAN AND J. VLČEK, Indefinitely preconditioned inexact Newton method for large sparse equality constrained non-linear programming problems, Numer. Linear Algebr. Appl., 5 (1998), pp. 219-247.

[47] K.-A. Mardal and R. Winther, Preconditioning discretizations of systems of partial differential equations, Numer. Linear Algebra Appl., 18 (2011), pp. 1-40.

[48] B. Mohar, The Laplacian spectrum of graphs, in Graph Theory, Combinatorics, and Applications. Kalamazoo, MI, 1988, Wiley, 1991, pp. 871-898.

[49] M. F. Murphy, G. H. Golub, and A. J. Wathen, A note on preconditioning for indefinite linear systems, SIAM J. Sci. Comput., 21 (2000), pp. 1969-1972.

[50] J. Nocedal And S. J. Wright, Numerical Optimization, Springer, New York, NY, 1999.

[51] C. C. Paige And M. A. Saunders, Solution of sparse indefinite systems of linear equations, SIAM J. Numer. Anal., 12 (1975), pp. 617-629.

[52] I. Perugia and V. Simoncini, Block-diagonal and indefinite symmetric preconditioners for mixed finite element formulations, Numer. Linear Algebra Appl., 7 (2000), pp. 585-616.

[53] I. Perugia, V. Simoncini, And M. Arioli, Linear algebra methods in a mixed approximation of magnetostatic problems, SIAM J. Sci. Comput., 21 (1999), pp. 1085-1101.

[54] J. Rommes AND W. H. A. SCHILDERS, Efficient methods for large resistor networks, IEEE Trans. Comput.-Aided Design of Integrated Circuits and Systems, 29 (2010), pp. 28-39.

[55] T. Rusten and R. Winther, A preconditioned iterative method for saddlepoint problems, SIAM J. Matrix Anal. Appl., 13 (1992), pp. 887-904.

[56] Y. SAAD, Krylov subspace methods for solving large unsymmetric linear systems, Math. Comp, 37 (1981), pp. 105-126.

[57] J. SchuR, Über Potenzreihen, die im Innern des Einheitskreises beschränkt sind, J. Reine Agnew. Math., 147 (1917), pp. 205-232.

[58] D. Silvester, H. Elman, and A. Ramage, Incompressible Flow and Iterative Solver Software (IFISS) version 3.3, October 2013. http://www.manchester.ac.uk/ifiss/.

[59] D. Silvester and A. Wathen, Fast iterative solution of stabilised Stokes sytems Part II: Using general block preconditioners, SIAM J. Numer. Anal., 31 (1994), pp. 1352-1367.

[60] D. J. Silvester And M. D. Minajlović, A black-box multigrid preconditioner for the biharmonic equation, BIT, 44 (2004), pp. 151-163.

[61] D. J. Silvester and V. Simoncini, An optimal iterative solver for symmetric indefinite systems stemming from mixed approximation, ACM Trans. Math. Software, 37 (2011), p. Article 42.

[62] V. Simoncini And M. Benzi, Spectral properties of the Hermitian and skew-Hermitian splitting preconditioner for saddle point problems, SIAM J. Matrix Anal. Appl., 26 (2004), pp. $377-$ 389. 
[63] I. H. Sloan And A. Sommariva, Approximation on the sphere using radial basis functions plus polynomials, Adv. Comput. Math., 29 (2008), pp. 147-177.

[64] I. H. Sloan AND H. Wendland, Inf-sup condition for spherical polynomials and radial basis functions on spheres, Math. Comp., 78 (2009), pp. 1319-1331.

[65] G. Strang, Introduction to Applied Mathematics, Wellesley-Cambridge Press, Wellesley, MA, 1986.

[66] — - A framework for equilibrium equations, SIAM Rev., 30 (1988), pp. 283-297.

[67] H. A. VAN DER VORST, Bi-CGSTAB: A fast and smoothly converging variant of BiCG for the solution of nonsymmetric linear systems, SIAM J. Sci. Statist. Comput., 13 (1992), pp. 631-644.

[68] P. S. Vassilevski, Multilevel Block Factorization Preconditioners: Matrix-based Analysis and Algorithms for Solving Finite Element Equations, Springer, New York, NY, 2008.

[69] P. S. Vassilevski and J. Wang, Multilevel iterative methods for mixed finite element discretizations of elliptic problems, Numer. Math., 63 (1992), pp. 503-520.

[70] M. vON GolitscheK AND W. A. Light, Interpolation by polynomials and radial basis functions on spheres, Constr. Approx., 17 (2001), pp. 1-18.

[71] A. Wathen, Preconditioning and convergence in the right norm, Int. J. Comput. Math, 84 (2007), pp. 1199-1209.

[72] A. Wathen, B. Fischer, And D. Silvester, The convergence rate of the minimal residual method for the Stokes problem, Numer. Math., 71 (1995), pp. 121-134.

[73] A. Wathen And T. ReEs, Chebyshev semi-iteration in preconditioning for problems including the mass matrix, Elec. Trans. Numer. Anal., 34 (2009), pp. 125-135.

[74] A. Wathen And D. Silvester, Fast iterative solution of stabilised Stokes systems. Part I: Using simple diagonal preconditioners, SIAM J. Numer. Anal., 30 (1993), pp. 630-649.

[75] A. J. Wathen, Realistic eigenvalue bounds for the Galerkin mass matrix, IMA J. Numer. Anal., 7 (1987), pp. 449-457.

[76] A. J. Wathen, B. Fischer, And D. J. Silvester, The convergence of iterative solution methods for symmetric and indefinite linear systems, in Numerical analysis 1997 (Dundee), D. F. Griffiths, D. J. Higham, and G. A. Watson, eds., Longman Harlow, 1998, pp. 230-243.

[77] M. H. WRIGHT, Interior methods for constrained optimization, Acta Numer., 1 (1992), pp. 341407.

[78] S. J. Wright, Primal-Dual Interior Point Methods, SIAM, Philadelphia, PA, 1997.

[79] W. Zulehner, Nonstandard norms and robust estimates for saddle point problems, SIAM J. Matrix Anal. Appl., 32 (2011), pp. 536-560. 